\title{
Renal denervation in patients with symptomatic chronic heart failure despite resynchronization therapy - a pilot study
}

\author{
Tomasz Drożdż, Marek Jastrzębski, Paweł Moskal, Aleksander Kusiak, Agnieszka Bednarek, \\ Katarzyna Styczkiewicz, Piotr Jankowski, Danuta Czarnecka
}

First Department of Cardiology, Interventional Electrocardiology and Hypertension, Jagiellonian University Medical College, Krakow, Poland

Adv Interv Cardiol 2019; 15, 2 (56): 240-246

DOI: https://doi.org/10.5114/aic.2019.86017

\begin{abstract}
A bstract
Introduction: Renal denervation (RD) has been shown to decrease sympathetic function in patients with hypertension. Its efficacy in symptomatic chronic heart failure (CHF) patients not responding to cardiac resynchronization therapy (CRT) has not been evaluated.

Aim: To assess whether a less invasive treatment method - renal denervation - is safe in symptomatic heart failure patients despite optimal medical treatment and resynchronization therapy and whether it is associated with an improvement in clinical status, exercise capacity and hemodynamic parameters.

Material and methods: The study was an open-label, randomized, controlled clinical trial. Patients were divided into an intervention (RD) and a control group. Clinical data collection, blood pressure (BP) measurements, echocardiography, 6-minute walk test (6MWT) and laboratory tests were performed before, 6 and 12 months after RD. The patients were followed-up to 24 months.

Results: We included 20 patients aged 52.0 to 86.0 years (median age: 71.5 years), 15 males and 5 females with median left ventricular ejection fraction (LVEF) of $32.5 \%$, body mass index $31.3 \mathrm{~kg} / \mathrm{m}^{2}$. Renal denervation was safe, no significant adverse effects were registered. There were no significant differences in LVEF, BP, 6MWT and N-terminal prohormone of brain natriuretic peptide (NT-proBNP) concentration 6 and 12 months after RD or control.

Conclusions: Our results indicate that RD in CHF patients not responding to CRT is safe and does not worsen exercise capacity and hemodynamic parameters.
\end{abstract}

Key words: chronic heart failure, cardiac resynchronization therapy, renal denervation.

S u m m a ry

Renal denervation (RD) in symptomatic chronic heart failure patients not responding to cardiac resynchronization therapy has not been evaluated. In our patients RD was safe, no serious adverse effects were registered. We detected no significant relation between renal artery denervation and clinical status, exercise capacity and hemodynamic parameters in optimally treated heart failure patients with systolic blood pressure over $110 \mathrm{~mm} \mathrm{Hg}$. Renal denervation was a safe procedure in this population.

\section{Introduction}

Currently, due to advances in treatment, we observe an increase in life expectancy in patients with hypertension and coronary artery disease. These diseases are among the leading causes of heart failure development and, as a result of better survival rate and longer lifespan in these patients, chronic heart failure (CHF) is increasing in prevalence [1-3]. Despite the unquestionable progress in treatment of these predisposing diseases the mortality rate of CHF patients has only slightly improved in recent years [4]. Cardiac resynchronization therapy (CRT) - a method of heart stimulation - was introduced into the treatment of heart failure almost 20 years ago [5]. The CRT is used in symptomatic patients with advanced stages of heart failure, despite optimal pharmacological therapy, with accompanying intraventricular conduction block. It is one of the most promis-

\section{Corresponding author:}

Tomasz Drożdż MD, First Department of Cardiology, Interventional Electrocardiology and Hypertension, Jagiellonian University Medical College, 17 Kopernika St, 31-501 Krakow, Poland, phone: +48 1242473 00, fax: +48 12424 73 20, e-mail: tomek_drozdz@wp.pl

Received: 7.09.2018, accepted: 14.02.2019. 
ing therapies, with a confirmed beneficial effect on heart failure outcomes [6]. However, there still remains a group of patients in which CRT it is not effective. About one third of patients who receive CRT do not have a meaningful clinical improvement. The reasons for this are complex and still not fully understood [7]. To date in this group of patients only left ventricular assist devices and heart transplant remain possible therapeutic modalities, but the accessibility of these methods is still insufficient.

\section{Aim}

We aimed to assess whether a less invasive treatment method - renal denervation - is safe in symptomatic heart failure patients despite optimal medical treatment and resynchronization therapy and whether it is associated with an improvement in clinical status, exercise capacity and hemodynamic parameters.

\section{Material and methods}

\section{Study design}

The study was conducted as an open-label, prospective, randomized, controlled clinical trial where patients were assigned to an intervention group undergoing the renal denervation procedure and a control group with no intervention based on the results of coin toss. Peripheral blood pressure (BP) measurements, transthoracic echocardiography, 6-minute walk test (6MWT), renal function assessment and biochemistry were performed at baseline and after 6 and 12 months. The patients were followed-up to 24 months. Optimal pharmacotherapy according to current European Society of Cardiology (ESC) guidelines was kept constant throughout the study [1, 2]. The echocardiographic optimization of atrioventricular and interventricular delay in CRT was performed for each patient before the study.

\section{Ethics}

The study was performed in accordance with the 1975 Declaration of Helsinki for Human Research and approved by the Jagiellonian University Bioethical Committee. Patients were included only if they gave their informed consent. The study is registered in the ClinicalTrials.gov repository (id: NCT02329145).

\section{Study population}

Adult CHF patients who met the following criteria were enrolled in this study: NYHA class II-IV; implanted resynchronization pacemaker according to current European guidelines at least 6 months prior to inclusion; persistent symptoms, defined as a lack of improvement in subjective dyspnea or exercise tolerance, despite stable pharmacological treatment over the previous 4 weeks (with ACE inhibitor and $\beta$-blocker unless contraindicated) and optimal biventricular (BIV) pacing (no less than
98\% of paced QRS complexes); left ventricular ejection fraction (LVEF) equal to or lower than 35\% in echocardiographic assessment prior to CRT implantation; estimated glomerular filtration rate (eGFR according to Modification of Diet in Renal Disease (MDRD) formula $\geq 30 \mathrm{ml} / \mathrm{min} / 1.73 \mathrm{~m}^{2}$ ).

We excluded patients with renal artery anatomy not eligible for denervation ( $<4 \mathrm{~mm}$ diameter, $<20 \mathrm{~mm}$ in length) [8]; history of prior renal artery intervention; single functioning kidney; systolic BP < $110 \mathrm{~mm} \mathrm{Hg}$; acute coronary syndrome or cerebrovascular event within last 3 months; serious medical conditions which may adversely affect safety such as significant peripheral vascular disease, abdominal aortic aneurysm, bleeding disorders (thrombocytopenia, hemophilia, or significant anemia) or pregnancy.

\section{Renal denervation}

Patients in the treatment group underwent renal artery denervation performed by an endovascular catheter-based approach in order to disrupt renal sympathetic nerves. A Symplicity radiofrequency renal-denervation catheter (Medtronic) was used. The central artery tree and renal artery were accessed by the femoral artery. The catheter was connected to a radiofrequency (RF) generator and multiple RF applications were performed in order to disrupt renal sympathetic nerves. We performed up to six radiofrequency ablations of up to 2 min duration that were separated both longitudinally and rotationally within each renal artery. Renal evaluations using duplex scan were performed in order to exclude baseline renovascular abnormalities and possible complications of the procedure, the main one being renal artery stenosis [8].

\section{Peripheral blood pressure measurement}

Office BP measurement was performed according to standards of the Polish Society of Hypertension [9]. Twenty-four-hour peripheral blood pressure monitoring was performed with the SpaceLabs 90207 monitor (Redmond, WA, USA). BP measurements were performed daily every $15 \mathrm{~min}$ ( $6 \mathrm{a}$ a.m. to 22 p.m.) and every $30 \mathrm{~min}$ at night (22.00 p.m. to 6.00 a.m.). Based on the acquired recordings mean values for systolic, diastolic blood pressure was calculated for $24 \mathrm{~h}$, day and night time.

\section{Echocardiography}

Echocardiography was performed using the Vivid 7 Pro (General Electric, Fairfield, USA), with a $2.5 \mathrm{MHz}$ probe by a single experienced operator who was blinded to the patients' allocation to experimental groups. Measurements were assessed according to the European Society of Echocardiography standards [10]. Left ventricle mass was calculated using the Devereux formula [10]. 


\section{Six-minute walk test (6MWT)}

The 6-minute walk test was performed in patients after a 10-minute, seated rest period. Patients were asked to march at their own pace, on a flat and level surface in an empty corridor. Patients were informed about the progress of the test on a regular basis; at the end of the 6-minute period, the total distance walked was measured. At baseline and after the test blood pressure and oxygen saturation were also measured [11].

\section{Biochemistry}

Biochemical parameters such as serum N-terminal prohormone of brain natriuretic peptide (NT-proBNP), creatinine (with the calculation of eGFR) and electrolytes were measured.

\section{End points of the study}

Primary end points were: 1) number of hospitalizations due to heart failure worsening within 1 year; 2) change in NYHA class within 1 year; 3) change in 6-minute walk test distance within 1 year. Additionally we evaluated the change in LVEF, BP values and NT-proBNP concentrations.

\section{Safety}

Safety parameters included death, stroke, myocardial infarction, renal artery stenosis or dissection, pseudoaneurysm at the femoral access site, bleeding, and reduction in eGFR.

\section{Statistical analysis}

All data were analyzed using the Statistica PL v.12.0 software. Categorical variables are reported as percentages and continuous variables as median and interquartile ranges when data distribution differed from the normal. The $\chi^{2}$ tests were applied to all categorical variables. For continuous variables, the Mann-Whitney test, the $t$-test for paired samples or the Wilcoxon matched pairs test was used. Effects for which the $p$-value was lower than the assumed level of significance $\alpha=0.05(p<0.05)$ were considered significant.

\section{Results}

\section{Patient cohort}

We screened 24 patients for being eligible for inclusion. In 4 patients in abdominal computed tomography we found tumors with suspicion of malignancy. These patients were excluded from the study [12]. Twenty patients aged 52.0 to 86.0 years (median age: 71.5 years), 15 males and 5 females with median LVEF of $32.5 \%$, in NYHA class II and III, median body mass index (BMI) $31.3 \mathrm{~kg} / \mathrm{m}^{2}$ were randomized. Secondary forms of cardiomyopathy were the most prevalent causes of $\mathrm{CHF}$ $12(60 \%)$ patients had ischemic cardiomyopathy. Eight
(40\%) patients had dilated cardiomyopathy of unknown etiology. The median QRS complex duration prior to CRT implantation was $150 \mathrm{~ms}$ and after the procedure 120 ms. Nineteen (95\%) patients had left bundle branch block morphology and 1 patient had right bundle branch block. The median LVEF prior to CRT implantation was $27 \%$. The clinical characteristics of enrolled patients are shown in Table I separately for the intervention and control groups. There were no significant differences in baseline characteristics according to group assignment.

The mean total time of denervation was $661 \pm 65 \mathrm{~s}$ for the right and $668 \pm 84 \mathrm{~s}$ for the left renal artery. The reduction of tissue resistance during ablation was 14.36 \pm 2.53 and $13.89 \pm 1.89 \%$, respectively.

\section{Primary endpoints}

During 12 months of observation 3 patients in the RD group and 2 in the control group were rehospitalized due to decompesation of heart failure ( $p=N S$ ) whereas during 24 months of observation 4 patients inthe RD and 5 patients incontrol group were rehospitalized ( $p=\mathrm{NS}$ ).

In the intervention group during 12 months of observation two patients improved from NYHA class III to II. In the control in 12 months 1 patient deteriorated from class II to III. The changes in NYHA class are presented in Figures 1 and 2.

The median change in 6MWT distance in the RD group was bigger compared to the control group, but it did not reach statistical significance $(+38.0(-50.0 ;+90.0)$ vs. $0.0(-100.0 ;+40.0) \mathrm{m}, p=0.212)$.

\section{Secondary endpoints}

Table II reports the changes in main clinical variables 6 and 12 months after renal denervation or allocation to the control group. We did not observe any significant changes in median heart rate, office blood pressure values, 6MWT distance or NT-proBNP concentrations.

\section{Safety results}

Renal denervation was safe, the procedure was done without complications in all of the patients and no significant periprocedural adverse effects (e.g. renal artery stenosis or dissection, pseudoaneurysm at the femoral access site, bleeding) were observed. After 6 and 12 months of observation we did not observe significant changes in eGFR in either the intervention or the control group. No patient developed renal-related symptoms.

In 2-year follow-up $1(5 \%)$ patient from the intervention group (10\%) died of an unknown cause. What could have influenced the death is the fact that he discontinued the vitamin $\mathrm{K}$ antagonist despite indications (persistent $\mathrm{AF}$ and self-contrast of blood in echocardiography). After the 2-year follow-up we received information about the second death of a patient from the intervention group. 
There were no strokes or myocardial infarctions during the course of the study.

\section{Discussion}

To the authors' knowledge this is the first report of a randomized controlled trial examining the role of renal denervation in patients with symptomatic heart failure with reduced ejection fraction not responding to resynchronization therapy. We found that the procedure of $\mathrm{RD}$ in patients with $\mathrm{CHF}$ is safe as no long-term adverse events resulted from the intervention. Especially, we did not observe any cases of renal artery stenosis or aneurysm. Furthermore, we observed no significant impairment of renal function according to the estimated glomerular filtration rate.
The activation of the sympathetic nervous system is one of the pathomechanisms responsible for the development and progression of heart failure. In chronic heart failure increased activity of the sympathetic and decreased activity of the parasympathetic system are present [13]. Based on previous studies involving renal denervation in patients with resistant hypertension, a decrease in the excessive activity of the sympathetic nervous system in heart failure patients after renal denervation might be expected [14]. This knowledge has generated an interest in renal denervation including catheter-based procedures as an attractive therapeutic approach in the therapy of resistant hypertension. Primary clinical data from two big trials - Symplicity HTN-1 and HTN-2 - demonstrated that catheter-based renal sympa-

Table I. Baseline characteristics of study participants

\begin{tabular}{|c|c|c|c|c|}
\hline Parameter & All $(n=20)$ & Denervation $(n=10)$ & Control $(n=10)$ & $P$-value \\
\hline NYHA Class & $3.0(2.0-3.0)$ & $3.0(3.0-3.0)$ & $2.5(2.0-3.0)$ & NS \\
\hline Age [years] & $71.5(67.5-78.0)$ & $75.0(65.0-81.0)$ & $71.0(70.0-76.0)$ & NS \\
\hline Sex, male, $n(\%)$ & $15(75)$ & $8(80)$ & $7(70)$ & NS \\
\hline $\mathrm{BMI}\left[\mathrm{kg} / \mathrm{m}^{2}\right]$ & $31.3(29.2-35.6)$ & $31.1(29.5-32.0)$ & $32.1(26.2-37.7)$ & NS \\
\hline LVEF prior to CRT (\%) & $27.0(22.0-33.5)$ & $27.5(22.0-35.0)$ & $27.0(25.0-32.0)$ & NS \\
\hline LVEF at RD (\%) & $32.5(27.5-37.5)$ & $33.5(30.0-40.0)$ & $32.0(25.0-36.0)$ & NS \\
\hline Heart rate [/min] & $70(63.0-75.0)$ & $64.0(61.0-75.0)$ & $70.0(64.0-75.0)$ & NS \\
\hline Office SBP [mm Hg] & $125(120-137)$ & $124(120-130)$ & $132(120-153)$ & NS \\
\hline Office DBP [mm Hg] & $74(70-80)$ & $73(70-80)$ & $74(70-78)$ & NS \\
\hline $24 \mathrm{~h} \mathrm{SBP}[\mathrm{mm} \mathrm{Hg}]$ & 111 (102-118) & $113(100-118)$ & $111(102-119)$ & NS \\
\hline $24 \mathrm{~h} \mathrm{DBP}[\mathrm{mm} \mathrm{Hg}]$ & $63(55-68)$ & $59(56-68)$ & $65(54-69)$ & NS \\
\hline 6MWT distance $[\mathrm{m}]$ & $330(270-415)$ & $320(240-330)$ & $380(300-440)$ & NS \\
\hline NT-proBNP [pg/ml] & $1116(491-2132)$ & $1116(445-1878)$ & $1148(537-2387)$ & NS \\
\hline $\mathrm{HbA}_{1 \mathrm{c}}(\%)$ & $6.1(5.8-6.4)$ & $6.3(6.0-6.5)$ & $5.8(5.6-6.4)$ & NS \\
\hline Infarction, $n$ (\%) & $8(40)$ & $5(50)$ & $3(30)$ & NS \\
\hline Diabetes, $n(\%)$ & $9(45)$ & $5(50)$ & $4(40)$ & NS \\
\hline Hypertension, $n(\%)$ & $15(75)$ & $7(70)$ & $8(80)$ & NS \\
\hline History of stroke, $n(\%)$ & $3(15)$ & $1(10)$ & $2(20)$ & NS \\
\hline Hypercholesterolemia, $n$ (\%) & $17(85)$ & $8(40)$ & $9(45)$ & NS \\
\hline CKD, $n(\%)$ & $6(30)$ & $4(40)$ & $2(20)$ & NS \\
\hline CABG, $n(\%)$ & $1(5)$ & $0(0)$ & $1(10)$ & NS \\
\hline $\mathrm{PCl}, n(\%)$ & $4(20)$ & $4(40)$ & $0(0)$ & NS \\
\hline $\mathrm{AF}, n(\%)$ & $16(80)$ & $8(80)$ & $8(80)$ & NS \\
\hline$\beta$-Blocker, $n(\%)$ & $20(100)$ & $10(100)$ & $10(100)$ & NS \\
\hline ACEI/ARB, $n(\%)$ & $17(85)$ & $8(80)$ & $9(90)$ & NS \\
\hline MRA, $n(\%)$ & $17(85)$ & $8(80)$ & $9(90)$ & NS \\
\hline Diuretic, $n(\%)$ & $19(95)$ & $9(90)$ & $10(100)$ & NS \\
\hline Digoxin, $n(\%)$ & $5(25)$ & $1(10)$ & $40(40)$ & NS \\
\hline
\end{tabular}

Values presented as median (interquartile range) or percentage. BMI - body mass index, LVEF - left ventricular ejection fraction, CRT - cardiac resynchronization therapy, $R D$ - renal denervation, SBP - systolic blood pressure, DBP - diastolic blood pressure, 6MWT-6-minute walk test, NT-proBNP - N-terminal prohormone of brain natriuretic peptide, $H b A_{1 c}$ - glycated hemoglobin, CKD - chronic kidney disease, CABG - coronary artery bypass graft, $P C l$ - percutaneous coronary intervention, AF - atrial fibrillation, ACEI - angiotensin-converting enzyme inhibitor, ARB - angiotensin receptor blocker, MRA - mineralocorticoid receptor antagonist, NYHA - NeW York Heart Association. 


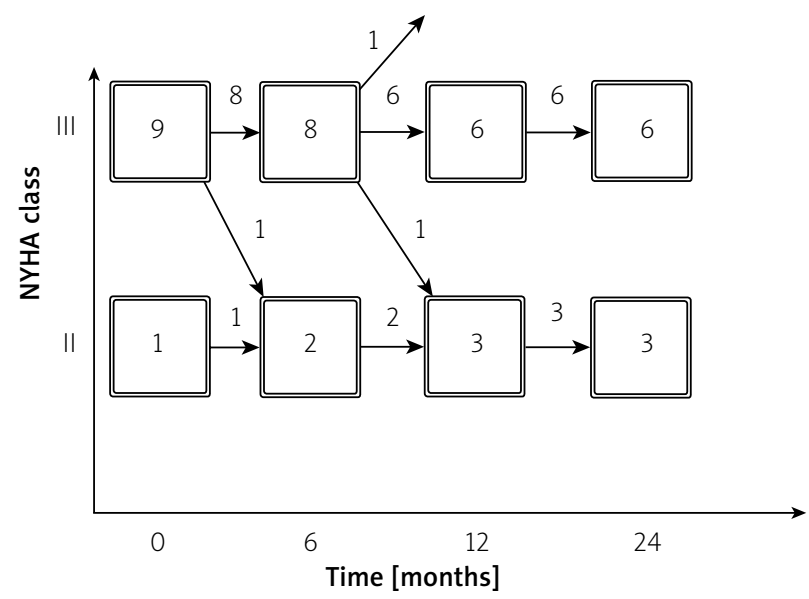

Figure 1. Changes in New York Heart Association (NYHA) class in the denervation group

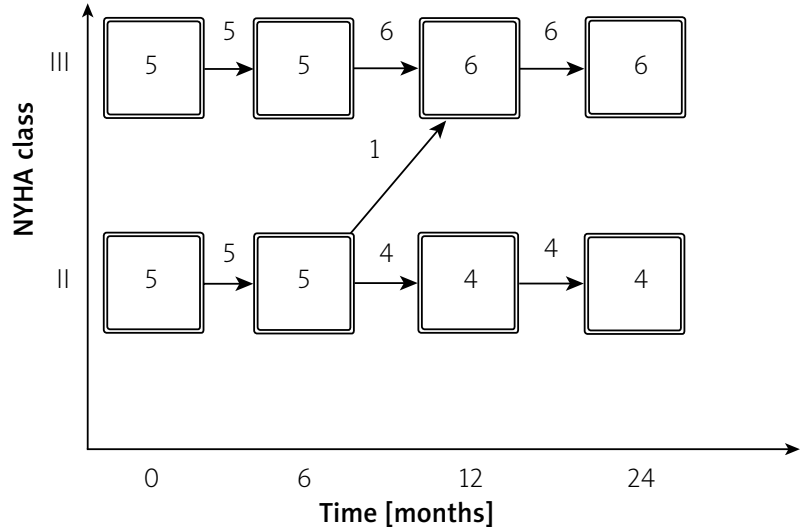

Figure 2. Changes in New York Heart Association (NYHA) class in the control group

Table II. Change in clinical variables at baseline and after 6 and 12 months

\begin{tabular}{|c|c|c|c|c|c|c|}
\hline \multirow[t]{2}{*}{ Parameter } & \multicolumn{3}{|c|}{ From baseline to $6^{\text {th }}$ month } & \multicolumn{3}{|c|}{ From baseline to $12^{\text {th }}$ month } \\
\hline & $\begin{array}{c}\mathrm{RD} \\
(n=10)\end{array}$ & $\begin{array}{l}\text { Control } \\
(n=10)\end{array}$ & $P$-value & $\begin{array}{c}\text { RD } \\
(n=10)\end{array}$ & $\begin{array}{l}\text { Control } \\
(n=10)\end{array}$ & $P$-value \\
\hline LVEF (\%) & $0.0(0.0-3.0)$ & $2.0(0.0 ; 7.0)$ & NS & $0.0(-3.0 ; 7.0)$ & $2.0(-3.0 ; 8.0)$ & NS \\
\hline Heart rate [/min] & $-1.5(-4.0 ;-1)$ & $1.0(0.0-5.0)$ & NS & $1.0(-3.0 ; 4.0)$ & $4.5(2.0 ; 6.0)$ & NS \\
\hline Office SBP [mm Hg] & $-1.0(-4.0 ; 20.0)$ & $0.0(-13.0 ; 15)$ & NS & $17.5(10.0-28.0)$ & $-5.0(-16.0 ; 22.0)$ & NS \\
\hline Office DBP [mm Hg] & $0.0(-2.0 ; 14.0)$ & $0.0(-5.0 ; 3.0)$ & NS & $8.0(2.0-18.0)$ & $1.5(-1.0 ; 5.0)$ & NS \\
\hline $24 \mathrm{~h} \mathrm{SBP}[\mathrm{mm} \mathrm{Hg}]$ & $3.0(-9.0 ; 15.0)$ & $-9.0(-15.0 ; 5.0)$ & NS & $7.5(6.0 ; 17.0)$ & $-1.5(-8.0 ; 3.0)$ & NS \\
\hline $24 \mathrm{~h} \mathrm{DBP}[\mathrm{mm} \mathrm{Hg}]$ & $5.0(-1.0 ; 9.0)$ & $-3.0(-9.0 ; 7.0)$ & NS & $4.5(3.0 ; 10.0)$ & $2.5(-6.0 ; 6.0)$ & NS \\
\hline 6MWT distance $[\mathrm{m}]$ & $20.0(0.0 ; 90.0)$ & $2.0(-20.0 ; 60.0)$ & NS & $38.0(-50.0 ; 90.0)$ & $0.0(-100.0 ; 40.0)$ & NS \\
\hline NT-proBNP [pg/ml] & $31(-450 ; 906)$ & $-69(-347 ; 48)$ & NS & $-11(-1059 ; 462)$ & $211(-22 ; 735)$ & NS \\
\hline eGFR $\left[\mathrm{ml} / \mathrm{min} / 1.73 \mathrm{~m}^{2}\right]$ & $0.70(-6.0 ; 17.1)$ & $-0.6(-11.2 ; 14.8)$ & NS & $-4.1(7.9 ; 0.0)$ & $0.4(-7.4 ; 6.7)$ & NS \\
\hline
\end{tabular}

Values presented as median (interquartile range). RD - renal denervation, LVEF-left ventricular ejection fraction, SBP-systolic blood pressure, DBP-diastolic blood pressure, 6MWT - 6-minute walk test, eGFR - estimated glomerular filtration rate.

thetic denervation can be safely used to reduce blood pressure within 24 months' observation $[15,16]$. However, the results of the Symplicity HTN-3 trial have not confirmed the blood-lowering effect of renal denervation [17]. However, several factors had a substantial impact on the results of the HTN-3 trial [18]. Later studies, like the multicenter, international, single-blind, randomized, sham-controlled SPYRAL HTN-OFF MED study showed in 80 patients a significant reduction in both office and 24-h ambulatory blood pressure values in the intervention group with no effect in the control group. There were no major adverse events in either group [19].

Renal denervation studies in hypertension have shown a positive effect of RD on cardiac remodeling such as a reduction in left atrial volume index [20], reduction in mean interventricular septum thickness, LV mass index (LVMI) and LV filling pressures and an increase in LVEF [21 22].

There have been several studies that evaluated the effect of renal denervation in patients with CHF. One such study is the REACH study, which showed that RD in patients with CHF is safe. No episodes of syncope, hypotension or other significant hemodynamic dysfunction were recorded during the acute phase after $\mathrm{RD}$ and renal function remained stable. Furthermore, 6 months after RD a non-significant blood pressure reduction and an improvement in 6-minute walk distance $(\Delta=27.1 \pm 9.7 \mathrm{~m}, p=0.03)$ were observed [23]. According to current guidelines, 6MWT is easy to administer and provides strong indications for measuring the response to medical intervention in patients with heart failure [11]. In ambulatory patients with systolic heart failure, $6 \mathrm{MWT}$ provides prognostic utility comparable to cardiopulmonary exercise tests, which is the gold standard for the assessment of exercise capacity in this group of patients [24]. In our study we found a non-significant improvement in 6MWT in patients 6 months after RD (320 (240; 330) vs. $335(280 ; 360) \mathrm{m}, p=\mathrm{NS})$.

In a randomized, controlled pilot study by Chen et al. 60 symptomatic patients with $\mathrm{CHF}$ and reduced 
EF (<40\%) were randomized to RD plus optimal medical therapy $(\mathrm{OMT})$ or only OMT. Throughout the study no severe adverse events were observed. Blood pressure was stable in both groups. Patients 6 months after RD showed a significant improvement in LVEF (31.1 \pm 5.7 vs. $41.9 \pm 7.9 \%, p<0.001)$ and 6 -minute walk test distance $(285.5 \pm 84.3$ vs. $374.9 \pm 91.9 \mathrm{~m}, p=0.043)$ and quality of life. NT-proBNP after RD was significantly reduced $(p<0.001)$. No significant changes in estimated glomerular filtration and no renal complications were found [25]. Compared to our study, the patients examined by Chen et al. were significantly younger, with less prevalent ischemic etiology and no stimulation.

Gao et al. in a study on 14 patients with CHF and ejection fraction $<45 \%$, who received bilateral RD, observed, 6 months after the procedure, a significant increase in 6MWT distance (152.9 \pm 38.0 vs. $334.3 \pm 94.4 \mathrm{~m}, p<$ $0.001)$ and $\mathrm{EF}(36.0 \pm 4.1$ vs. $43.8 \pm 7.9 \%, p=0.003)$. On the other hand, systolic BP decreased from $138.6 \pm 22.1$ to $123.2 \pm 10.5 \mathrm{~mm} \mathrm{Hg}(p=0.026)$ and diastolic BP from $81.1 \pm 11.3$ to $72.9 \pm 7.5 \mathrm{~mm} \mathrm{Hg}(p=0.032)$. The authors did not observe adverse results or worsening of renal function. However, one of the limitations of this study was the lack of a control group [26].

The RDT-PEF study aimed at assessing the effect of RD with the Symplicity catheter vs standard therapy on several clinical parameters (Minnesota Living with Heart Failure Questionnaire score, peak oxygen uptake $\left(\mathrm{VO}_{2}\right)$ on exercise, BNP, E/e', LA volume index or LV mass index). Due to recruitment difficulties, the study was terminated after inclusion of only 25 patients and was underpowered to detect whether RD influenced the defined endpoints. Changes in eGFR were comparable in the two groups, but 2 patients required balloon angioplasty during the RD to treat renal artery wall edema [27].

In a study by Hopper et al. on $40 \mathrm{HF}$ patients RD was associated with small reductions in NT-proBNP and 120-minute glucose tolerance at 12 months with no RD-associated adverse effect, except for 1 case of renal artery occlusion [28].

These previously described beneficial effects of RD on $\mathrm{BP}$ may also extend to the preservation of renal function. Kiuchi et al. reported beneficial effects of RD in 24 patients with chronic kidney disease and refractory hypertension on kidney function. A significant improvement in eGFR (85.4 \pm 34.9 vs. $\left.64.4 \pm 23.9 \mathrm{ml} / \mathrm{min} / 1.73 \mathrm{~m}^{2}, p<0.0001\right)$ and a decrease in the median urine albumin/creatinine ratio (15.7 (10.3-34.2) vs. $48.5(35.8-157.2) \mathrm{mg} / \mathrm{g}, p=$ $0.0017)$ at 6 months of follow-up were present [29]. There were also reports of a reduction in albuminuria after RD in patients with resistant hypertension [30]. Furthermore, a reduction in Doppler sonographic renal resistive index, which reflects systemic and renal hemodynamics, has been associated with progression of renal impairment and which could be a potential noninvasive predictor of lack of response to RD, has been observed [31]. In our study we observed a non-significant increase in eGFR after RD proving its safety in CHF patients in regard to renal function. Unfortunately we did not evaluate microalbuminuria.

Throughout the study one of the patients from the intervention group died of an unknown cause at home. We could not associate the death with the procedure of RD, as no acute and follow-up disturbances have been noted. The studied population consisted of patients with severe heart failure that did not respond to optimal pharmacoand electrotherapy and the worsening of the underlying disease was the most probable cause of death.

Our study has several strengths. Firstly, it is the first study regarding renal denervation in patients with CHF not responding to CRT. To date, there have been several ongoing studies concerning RD in CHF, but there are no studies evaluating this intervention in severe stages of CHF that do not benefit from CRT. So far in this scientific area only limited data are available. Thus the above study has a pilot character.

There are also a few limitations. First, the small number of patients may have resulted in the study being underpowered to show significant change in evaluated parameters. One may speculate that RD could counteract the progression of CHF in these patients. Thus, the observed improvement in exercise capacity was small and did not reach statistical significance. Second, the use of a single-electrode catheter might have led to an inability to create a circumferential lesion effortlessly, which unfortunately cannot reliably be assessed during the procedure. In a 2017 position paper on the autonomic nervous system as a therapeutic target in heart failure from the Translational Research Committee of the Heart Failure Association of the ESC the use of such catheters is discouraged. However, when the protocol of this study was created and introduced, newer catheters and the aforementioned data were not yet available [32]. Furthermore, despite the increase in CRT implantation in recent years, the population of patients with this device who are eligible for RD is still limited due to hypotension or atherosclerosis of renal arteries. The lack of a sham procedure also limits the power of the results.

\section{Conclusions}

We detected no significant relation between renal artery denervation and clinical status, exercise capacity and hemodynamic parameters in optimally treated heart failure patients with systolic blood pressure over $110 \mathrm{~mm} \mathrm{Hg}$. Renal denervation was a safe procedure in this population.

\section{Acknowledgments}

The study was supported by a grant of the National Science Centre (NSC) number UMO-2012/07/13/NZ5/ 02613. 


\section{Conflict of interest}

The authors declare no conflict of interest.

\section{References}

1. McMurray JJ, Adamopoulos S, Anker SD, et al. ESC guidelines for the diagnosis and treatment of acute and chronic heart failure 2012. Eur J Heart Fail 2012; 14: 803-69.

2. Ponikowski P, Voors AA, Anker SD, et al. 2016 ESC Guidelines for the diagnosis and treatment of acute and chronic heart failure: The Task Force for the diagnosis and treatment of acute and chronic heart failure of the European Society of Cardiology (ESC) developed with the special contribution of the Heart Failure Association (HFA) of the ESC. Eur Heart J 2016; 37: 2129-200.

3. Kawecka-Jaszcz K, Kloch-Badełek M, Wojciechowska W. Hypertension as a risk factor for heart failure. Arterial Hypertens 2011; 15: 275-82.

4. Chen J, Normand SL, Wang Y, Krumholz HM. National and regional trends in heart failure hospitalization and mortality rates for Medicare beneficiaries, 1998-2008. JAMA 2011; 306: 1669-78.

5. Leyva F, Nisam S, Auricchio A. 20 years of cardiac resynchronization therapy. J Am Coll Cardiol 2014; 64: 1047-58.

6. Cleland JG, Abraham WT, Linde C, et al. An individual patient meta-analysis of five randomized trials assessing the effects of cardiac resynchronization therapy on morbidity and mortality in patients with symptomatic heart failure. Eur Heart J 2013; 34: 3547-56.

7. Sohaib SM, Finegold JA, Nijjer SS, et al. Opportunity to increase life span in narrow QRS cardiac resynchronization therapy recipients by deactivating ventricular pacing: evidence from randomized controlled trials. JACC Heart Fail 2015; 3: 327-36.

8. Esler MD, Krum H, Sobotka PA, et al. Renal sympathetic denervation in patients with treatment-resistant hypertension (The Symplicity HTN-2 Trial): a randomised controlled trial. Lancet 2010; 376: 1903-9.

9. Tykarski A, Narkiewicz K, Gaciong Z, et al. 2015 guidelines for the management of hypertension. Arterial Hypertens 2015; 19: 53-83.

10. Lang RM, Bierig M, Devereux RB, et al. Recommendations for chamber quantification. Eur J Echocardiogr 2006; 7: 79-108.

11. ATS Committee on Proficiency Standards for Clinical Pulmonary Function Laboratories. ATS statement: guidelines for the six-minute walk test. Am J Respir Crit Care Med 2002; 166: 111-7.

12. Kusiak A, Bednarek A, Moskal P, et al. Should abdominal CT scan be performed routinely prior to CRT insertion? Przegl Lek 2016; 73: 821-3.

13. Kishi T. Heart failure as an autonomic nervous system dysfunction. J Cardiol 2012; 59: 117-22.

14. Krum H, Sobotka P, Mahfoud F, et al. Device-based antihypertensive therapy: therapeutic modulation of the autonomic nervous system. Circulation 2011; 123: 209-15.

15. Krum H, Schlaich MP, Sobotka PA, et al. Percutaneous renal denervation in patients with treatment-resistant hypertension: final 3-year report of the Symplicity HTN-1 study. Lancet 2014; 383: 622-9.

16. Esler MD, Böhm M, Sievert H, et al. Catheter-based renal denervation for treatment of patients with treatment-resistant hypertension: 36 month results from the SYMPLICITY HTN-2 randomized clinical trial. Eur Heart J 2014; 35: 1752-9.

17. Bhatt DL, Kandzari DE, O'Neill WW, et al. A controlled trial of renal denervation for resistant hypertension. N Engl J Med 2014; 370: 1393-401.
18. Kądziela J, Warchoł-Celińska E, Prejbisz A, et al. Renal denervation - can we press the "ON" button again? Adv Interv Cardiol 2018; 14: 321-7.

19. Townsend RR, Mahfoud F, Kandzari DE, et al. Catheter-based renal denervation in patients with uncontrolled hypertension in the absence of antihypertensive medications (SPYRAL HTN-OFF MED): a randomised, sham-controlled, proof-of-concept trial. Lancet 2017; 390: 2160-70.

20. Brandt MC, Mahfoud F, Reda S, et al. Renal sympathetic denervation reduces left ventricular hypertrophy and improves cardiac function in patients with resistant hypertension. J Am Coll Cardiol 2012; 59: 901-9.

21. Schirmer SH, Sayed MM, Reil JC, et al. Atrial remodeling following catheter-based renal denervation occurs in a blood pressure- and heart rate-independent manner. JACC Cardiovasc Interv 2015; 8: 972-80.

22. Mahfoud F, Urban D, Teller D, et al. Effect of renal denervation on left ventricular mass and function in patients with resistant hypertension: data from a multi-centre cardiovascular magnetic resonance imaging trial. Eur Heart J 2014; 35: 2224-31.

23. Davies JE, Manisty CH, Petraco R, et al. First-in-man safety evaluation of renal denervation for chronic systolic heart failure: primary outcome from REACH-Pilot study. Int J Cardiol 2013; 162: 189-92.

24. Forman DE, Fleg JL, Kitzman DW, et al. 6-min walk test provides prognostic utility comparable to cardiopulmonary exercise testing in ambulatory outpatients with systolic heart failure. J Am Coll Cardiol 2012; 60: 2653-61.

25. Chen W, Ling Z, Xu Y, et al. Preliminary effects of renal denervation with saline irrigated catheter on cardiac systolic function in patients with heart failure: a prospective, randomized, controlled, pilot study. Catheter Cardiovasc Interv 2017; 89: E153-61.

26. Gao JQ, Xie Y, Yang W, et al. Effects of percutaneous renal sympathetic denervation on cardiac function and exercise tolerance in patients with chronic heart failure. Rev Port Cardiol 2017; 36: 45-51.

27. Patel HC, Rosen SD, Hayward C, et al. Renal denervation in heart failure with preserved ejection fraction (RDT-PEF): a randomized controlled trial. Eur J Heart Fail 2016; 18: 703-12.

28. Hopper I, Gronda E, Hoppe UC, et al. Sympathetic response and outcomes following renal denervation in patients with chronic heart failure: 12 -month outcomes from the symplicity HF feasibility study. J Card Fail 2017; 23: 702-7.

29. Kiuchi MG, Maia GL, de Queiroz Carreira MA, et al. Effects of renal denervation with a standard irrigated cardiac ablation catheter on blood pressure and renal function in patients with chronic kidney disease and resistant hypertension. Eur Heart J 2013; 34: 2114-21.

30. Ott C, Mahfoud F, Schmid A, et al. Improvement of albuminuria after renal denervation. Int J Cardiol 2014; 173: 311-5.

31. Mahfoud F, Cremers B, Janker J, et al. Renal hemodynamics and renal function after catheter-based renal sympathetic denervation in patients with resistant hypertension. Hypertension 2012; 60: 419-24.

32. van Bilsen M, Patel HC, Bauersachs J, et al. The autonomic nervous system as a therapeutic target in heart failure: a scientific position statement from the Translational Research Committee of the Heart Failure Association of the European Society of Cardiology. Eur J Heart Fail 2017; 19: 1361-78. 